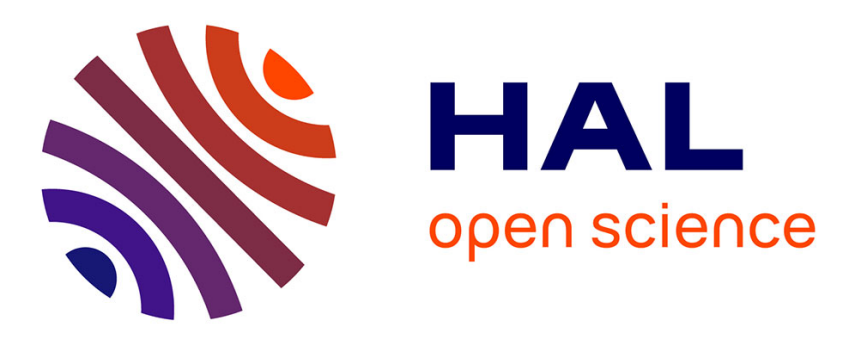

\title{
2D/3D Deep Registration for Real-Time Prostate Biopsy Navigation
}

Tamara Dupuy, Clément Beitone, Jocelyne Troccaz, Sandrine Voros

\section{To cite this version:}

Tamara Dupuy, Clément Beitone, Jocelyne Troccaz, Sandrine Voros. 2D/3D Deep Registration for Real-Time Prostate Biopsy Navigation. SPIE Medical Imaging 2021, SPIE, Feb 2021, Digital congress, United States. pp.115981P, 10.1117/12.2579874 . hal-03131349

\section{HAL Id: hal-03131349 \\ https://hal.science/hal-03131349}

Submitted on 4 Feb 2021

HAL is a multi-disciplinary open access archive for the deposit and dissemination of scientific research documents, whether they are published or not. The documents may come from teaching and research institutions in France or abroad, or from public or private research centers.
L'archive ouverte pluridisciplinaire HAL, est destinée au dépôt et à la diffusion de documents scientifiques de niveau recherche, publiés ou non, émanant des établissements d'enseignement et de recherche français ou étrangers, des laboratoires publics ou privés. 


\title{
2D/3D Deep Registration for Real-Time Prostate Biopsy Navigation
}

\author{
Tamara Dupuy $^{\mathrm{a}}$, Clément Beitone ${ }^{\mathrm{a}}$, Jocelyne Troccaz ${ }^{\mathrm{a}}$, and Sandrine Voros ${ }^{\mathrm{a}}$ \\ ${ }^{a}$ Univ. Grenoble Alpes, CNRS, Grenoble-INP, INSERM, TIMC-IMAG, 38000 Grenoble, \\ France
}

\begin{abstract}
The accuracy of biopsy sampling and the related tumor localization are major issues for prostate cancer diagnosis and therapy. However, the ability to navigate accurately to biopsy targets faces several difficulties coming from both transrectal ultrasound (TRUS) image guidance properties and prostate motion or deformation. To reduce inaccuracy and exam duration, the main objective of this study is to develop a real-time navigation assistance. The aim is to provide the current probe position and orientation with respect to the deformable organ and the next biopsy targets. We propose a deep learning real-time $2 \mathrm{D} / 3 \mathrm{D}$ registration method based on Convolutional Neural Networks (CNN) to localize the current 2D US image relative to the available 3D TRUS reference volume. We experiment several scenarii combining different input data including: pair of successive 2D US images, the optical flow between them and current probe tracking information. The main novelty of our study is to consider prior navigation trajectory information by introducing previous registration result. This model is evaluated on clinical data through simulated biopsy trajectories. The results highlight significant improvement by exploiting trajectory information especially through prior registration results and probe tracking parameters. With such trajectory information, we achieve an average registration error of $2.21 \mathrm{~mm} \pm 2.89$. The network demonstrates efficient generalization capabilities on new patients and new trajectories, which is promising for successful continuous tracking during biopsy procedure.
\end{abstract}

Keywords: Image Registration; Convolutional Neural Network; Image-guided Intervention; Prostate biopsy

\section{INTRODUCTION}

\subsection{Clinical context and motivations}

The accurate localization of targets and navigation towards them is critical to many clinical tasks especially for image-guided interventions on soft-tissue. 2D images are often used for intra-operative guidance and most often provide real-time information about the organs and the intervention, at the expense of a limited view of the $3 \mathrm{D}$ scene. On the other hand, preoperative 3D acquisitions usually include additional and better information for both visualization and navigation purposes. Therefore, the robust and real-time fusion of preoperative 3D image with intra-operative $2 \mathrm{D}$ image flow has a great potential for guidance, as it can cope with real-time anatomy deformation and provide a reliable estimate of the position of interventional targets.

A typical case is prostate biopsy, where navigation to biopsy targets faces several difficulties coming from both the transrectal ultrasound (TRUS) modality properties and prostate characteristics. Indeed, the real-time organ visualization is made difficult due to US modality which presents variable image quality (low signal to noise ratio, artefacts, speckle), poor sensitivity to cancerous tissue and limited 2D anatomical context. Moreover, the clinician's mental 3D representation is even more challenging due to the spherical, symmetrical and deformable characteristics of the prostate. The impossibility to figure out the effect of ultrasound probe motion on the prostate and related targets may make samples localization very uncertain. These difficulties may result in longer and uncomfortable examination procedure for the patient, but may also produce biased diagnosis and inappropriate therapeutic decision (about $30 \%$ of false negative and risk of overtreatment ${ }^{1,2}$ ). Finally, wrong sample localization may also result in inaccurate treatment when using focal therapy application.

Further author information: (E-mail) first.last@univ-grenoble-alpes.fr 
In this context where prostate cancer diagnosis and therapy are at stakes, providing real-time assistance during prostate biopsy interventions has led to several developments over the past two decades ${ }^{3,4}$.

First approaches focused on improving biopsy localization by using 3D/3D registration to include preoperative information. These approaches used either optimization-based ${ }^{5}$ or deep-learning based methods ${ }^{6-10}$. They register preoperative 3D MR reference image to 3D TRUS image, acquired at the start of the procedure. However, these approaches do not take into account intra-operative anatomical deformations and result in limited accuracy. Moreover, the initial 3D TRUS image is most often reconstructed from probe sweeping (2D slices and their position information), which results in a biased 3D volume (tissue deformation due to probe sweep). To include updated anatomical deformations, other studies ${ }^{11}$ propose to register the initial 3D TRUS image to 3D TRUS images acquired all along the procedure. However, they are computationally intensive and need long 3D acquisition time. Moreover, they are used only at static positions, just before getting each biopsy sample. Finally, even if this first kind of approaches enables a better localization of biopsy sampling, the clinicians must still rely only on limited 2D US flow and personal experience to navigate efficiently between two biopsy sites.

This has motivated another kind of approaches ${ }^{1,5,12-15}$ focused on providing real-time navigation assistance to the clinicians. They aim at computing the current position of the probe relatively to the next biopsy target. This dynamic assistance is based on real-time $2 \mathrm{D} / 3 \mathrm{D}$ registration which links the $2 \mathrm{D}$ live image to the initial $3 \mathrm{D}$ TRUS image. Optimization-based methods have still limited results in terms of accuracy ${ }^{15}$, computational efficiency $^{5,12,14}$, or clinical evaluation (limited data set or phantom) $)^{1,13}$. More recently, deep learning-based strategies provided a new opportunity to develop robust real-time guidance. Related work is described in more details in the next section.

Finally, registration results must satisfy clinical requirements both in terms of accuracy and computational efficiency. Regarding accuracy, a consensus value of 0.5 cc exists concerning the smallest significant treatable tumour $^{1,2}$ and corresponds approximately to a sphere having a radius of $5 \mathrm{~mm}$. Therefore, $5 \mathrm{~mm}$ will be used as an acceptance range to evaluate the registration quality. Moreover, for real-time navigation assistance, the computational requirement is related to the TRUS guidance frequency (about 10-20 Hz). A computation time below $50 \mathrm{~ms}$ would provide real-time guidance.

\section{$1.22 \mathrm{D} / 3 \mathrm{D}$ deep registration for real-time navigation assistance}

This section extends to any $2 \mathrm{D} / 3 \mathrm{D}$ real-time deep registration study considering, either slices location inside a volume, or volume reconstruction from slices. Most of the literature ${ }^{16-24}$ is focused on a supervised regression task, for end-to-end prediction of rigid transformation parameters only (rotations and translations) and most often uses convolutional neural networks (CNN) architectures. A majority of the methods predict slices localization with respect to a fixed reference volume by only inputting the moving slices. Few studies combined both $2 \mathrm{D}$ and $3 \mathrm{D}$ inputs in the same model ${ }^{25,26}$. Even if most of these studies aim to solve $2 \mathrm{D} / 3 \mathrm{D}$ registration, comparing them remains difficult, as data involved may be very different in terms of modality (mostly MRI, vs. US), source (clinical or phantom), motion generation (recorded or artificially simulated), datasets, and preprocessing (centered, initialized, segmented, etc.). Evaluation is also a crucial criterion for result interpretation. It depends also on the metrics used (mean squared error, target registration error, etc.), and transformation representation (quaternion, Euler angle, rotation matrix, fiducials, etc.).

We find several main structures among all these studies: (i) some use a hierarchical structure ${ }^{6,7,23-27}$. It allows a coarse-to-fine registration by using results from a previous step to initialize the next one. First steps rely on networks performing either segmentation tasks $6,7,23-25$, classification tasks ${ }^{26}$, or registration initializations $^{6,7,23,24}$. (ii) Others decompose the registration problem into sub problems with a more local focus ${ }^{8,23,24}$. It allows reducing the complexity of a problem and computation time, but also avoiding convergence towards local minima. Results of each sub problem are then averaged through either the calculation of the loss function ${ }^{8}$, or combined through concatenation on fully connected layer ${ }^{23,24}$. (iii) Some others convert the registration problem into a classification problem. Classification can be difficult to exploit as most of registration problem parameters are related to continuous and complex representations. Classification can solve partly ${ }^{26}$ or totally $^{21}$ the registration problem. (iv) Finally, a few studies focus on dynamical structures by using recurrent network. LSTM have shown their values to model a complex temporal process and can be used to register a complete sequence of slices ${ }^{22}$.

A promising work ${ }^{28}$, we want to describe in more details, proposes a CNN for 3D US volume reconstruction 
from 2D US images, by estimating relative transform parameters between successive US frames. The network main input is the pair of successive images. Additional inputs include the optical flow between the two images, and current probe tracking information. The authors showed that incorporating such additional information to the pair of images significantly helped the network to be more accurate and robust, much more than changes in its architecture. But even with extremely simple probe movements, results reveal a drift along the reconstruction due to error accumulation when performing successive registrations. Even if this work presents different objectives compared to $2 \mathrm{D} / 3 \mathrm{D}$ registration, it illustrates a promising method to correlate $2 \mathrm{D}$ information to global $3 \mathrm{D}$ localization using relative and successive information.

\subsection{Objectives and contribution}

To allow navigation assistance during biopsy procedures, the main objective of this study is to develop and evaluate a deep learning real-time $2 \mathrm{D} / 3 \mathrm{D}$ registration method to localize the "live" $2 \mathrm{D}$ US prostate image relative to the available 3D reference volume (e.g. preoperative US). Similarly to most of the studies found in literature, we focused on supervised CNN to predict rigid transformation parameters. The proposed method is mainly inspired from Prevost ${ }^{28}$ for their network architecture as well as their additional inputs strategies. The main novelty of the presented study is to consider prior navigation trajectory information. By introducing the registration result information of the previous 2D US image, we intend to help the network to better estimate the current slice localization. This information is added as additional input to the network and not as a conventional initialization step. The benefit of such information is evaluated through this study.

\section{METHOD}

Being given a series of $2 \mathrm{D}$ US images $\left(I_{1}, I_{2}, \ldots, I_{t}\right)$ acquired during a prostate biopsy exam, the aim is to register at each time $t$ the current image $I_{t}$ to the $3 \mathrm{D}$ US reference volume. The resulting $2 \mathrm{D} / 3 \mathrm{D}$ rigid transform is denoted as $T_{t}$. Due to data construction (see Part 3.1), only the 3 rotational parameters $(\theta x, \theta y, \theta z)$ are considered to describe the orientation of the slice with respect to the volume. They are represented using the Euler angle encoding.

Similarly to Prevost ${ }^{28}$, we include the following inputs : (i) Pair of images: the current slice $I_{t}$ to localize and the previous one $I_{t-1}$. The pair is fed into the network as a 2-channel image (cropped to 150x250 pixels), so that the spatial information between them is coupled from the very first layer through the network's convolutions. This input is common in all the scenarii. (ii) Optical flow (optional input): this 2D vector field represents the relative in-plane motion between the two images. The two components of this vector field are concatenated to the pair of images to form a 4-channel image input that preserves the spatial correspondence. As in Prevost ${ }^{28}$, the optical flow is based on Farnebäck algorithm ${ }^{29}$. (iii) Probe tracking information (optional input): the probe sensor information corresponding to the relative displacement between two successive images. We consider for relative displacement, again, only the rotation parameters that we concatenate to the 512 -vector layer of the network. We simulated those relative displacements using two successive global ones $\left(T_{t}^{-1} * T_{t-1}\right)$, to which we add a maximum random noise of $1^{\circ}$ corresponding to the average inertial sensor noise ${ }^{30}$.

We experiment 4 different scenarii by combining the proposed inputs: Scenario 1: only the pair of images, Scenario 2: pair of images + optical flow, Scenario 3: pair of images + probe tracking, Scenario 4: pair of images + optical flow + probe tracking.

Finally, to evaluate the contribution of prior trajectory information, another input $\left(T_{\text {prior }}\right)$ is added to each of these scenarii. This prior information corresponds to the previous registration result $T_{t-1}$. This rotation parameter label vector is added on the same 512-vector layer of the network.

\subsection{Network architecture and training}

The CNN architecture is presented in Figure 1. It is composed of 12 convolutional layers, all followed by a rectified linear unit (ReLU) and max pooling layers. Then, the network consists in 6 fully connected layers, with a decreasing number of neurons to finally connect with the final layer, the 3 parameters vector corresponding to the rotation part of the rigid transform we wish to estimate.

Training is performed in a supervised way by adjusting the network's parameters to minimize the mean squared error between the network predictions and the labels (ground truth rotation parameters of $T_{t}$ ). Among 


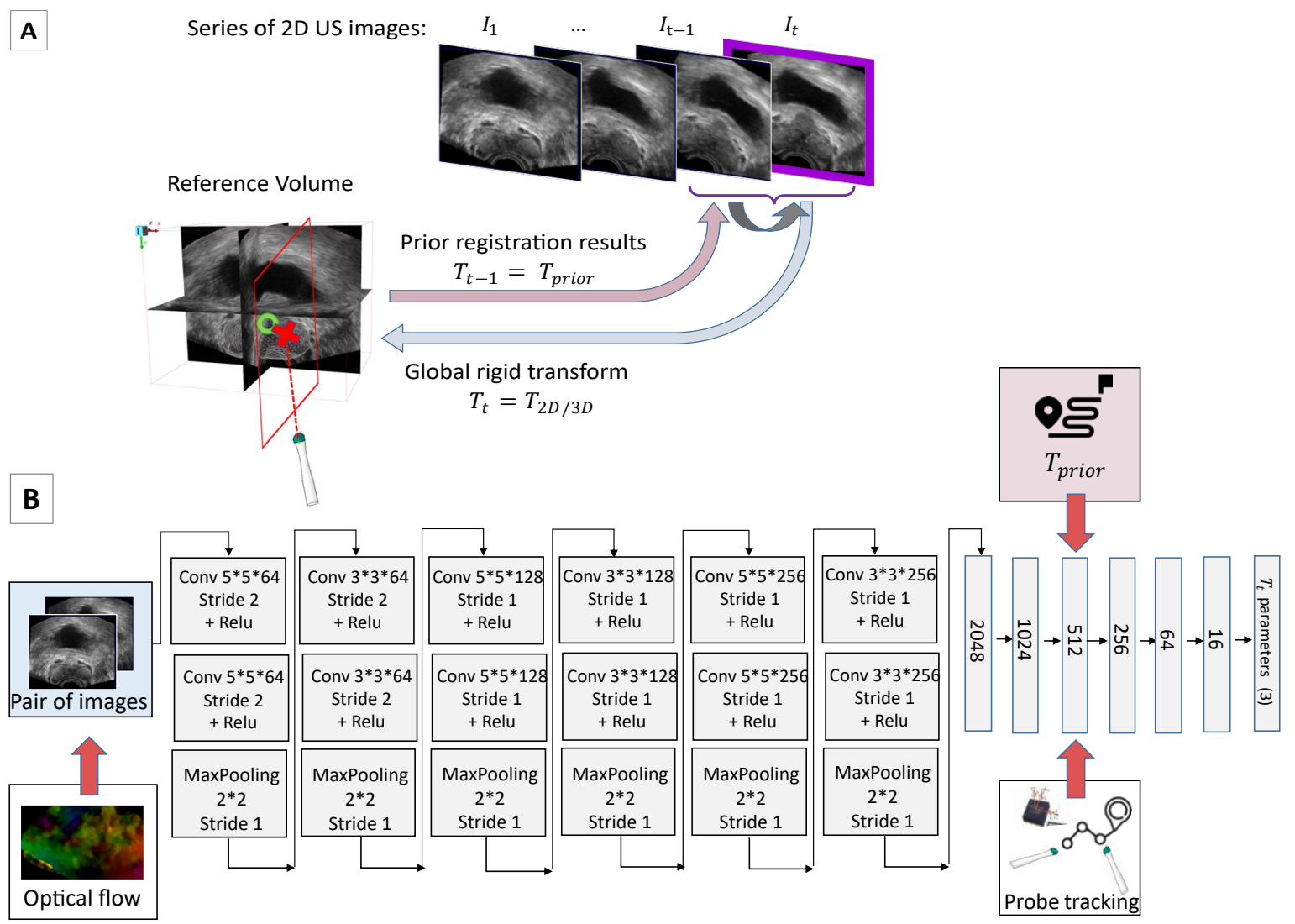

Figure 1. Method. A) Problem statement. The objective is to estimate the rigid transform parameters between a 2D US image and the reference volume in an end-to-end fashion, being given additional information such as previous registration prior, relative probe motion, and optical flow. B) Network Architecture

several optimization techniques, we achieved good performance with the Nadam optimizer. The weights of the network were all initialized with a Gaussian distribution (mean $=0, \mathrm{std}=0.01)$. We used large batch size (500) to achieve better generalization among our data to reach the global minima. The initial learning rate is $1 \mathrm{e}-4$ and the training is performed over 50 epochs, for which we obtain robust and rapid convergence. All these training parameters were mainly inspired from literature ${ }^{28}$ and improved experimentally. Our model is trained on a Titan RTX GPU using TensorFlow via Keras.

\section{EXPERIMENTS AND RESULTS}

\subsection{Data generation}

Capturing a series of 2D US images along real clinical procedures with their relative localization to the reference volume requires probe tracking. To avoid the complexity of such acquisition in clinical routine, linked to the regulatory challenges of integrating system calibration bricks in the operating room, we used a previously developed biopsy simulator (Biopsym) ${ }^{31,32}$ illustrated in Figure 2.

This simulator is composed of a US probe mock-up attached to a haptic device (Phantom Omni, Sensable Devices Inc.) which records the position and orientation of the probe at $100 \mathrm{~Hz}$. It gathers anonymized clinical data collected from real biopsy series, for which it provides for each patient: the 3D reference volume (e.g. preoperative US) and the associated prostate mesh. When a user moves the probe mock-up, the simulator generates a succession of 2D US images, resliced from the reference volume, and collects them as 2D image flow. Thus, each ground-truth slice localization with respect to the reference volume $\left(T_{t}\right)$ is known from the haptic device tracking. Each series of 2D US images is thus associated to a real biopsy trajectory. The process is summarized in Figure 2. 


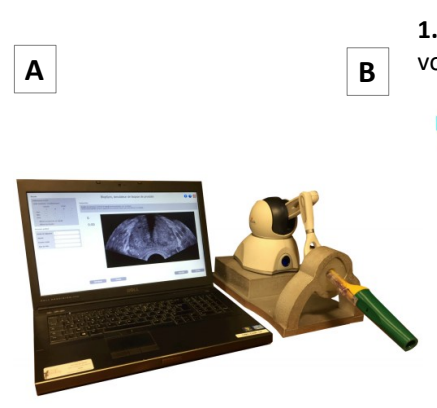

1. Simulator patient data :3D reference volume and associated prostate mesh

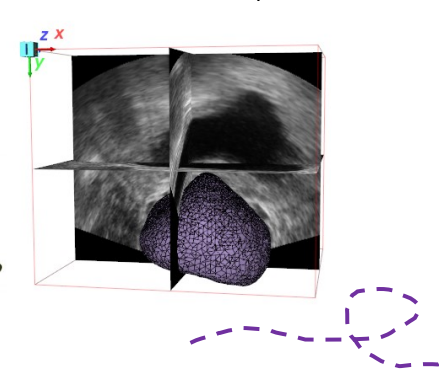

2. Recording of the position and orientation of the probe at a frequency of $100 \mathrm{~Hz}$
3. Extract the median slice from the reoriented reference volume

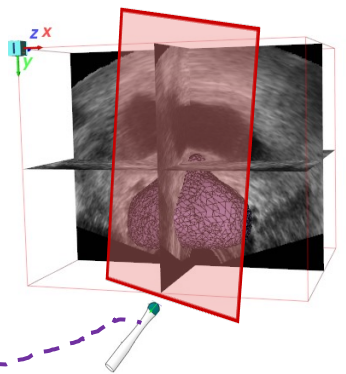
with respective ground-truth slice localization $\left(T_{t}\right)$

Figure 2. Data generation process. A) Biopsym Simulator. B) Generation of the 2D US image flow from a clinical reference volume, associated with a realistic biopsy trajectory.

\subsection{Datasets}

We asked a urologist to perform randomized biopsies (12 systematic biopsies spread homogeneously in different regions of the prostate) on the simulator. We recorded 7 trajectories that we applied to a large database composed of 70 US reference volumes from 70 different patients. These volumes were acquired during routine biopsy series with two different commercial US-based guidance systems (Urostation ${ }^{\circledR}$ and Trinity ${ }^{\circledR}$ from Koelis SAS), by clinicians from the Grenoble University Hospital. We took care to keep unknown trajectories and unknown patients for the testing set. To simulate a realistic acquisition frequency with significant motions between two slices, the time step between two successive images was set to $150 \mathrm{~ms}(6.6 \mathrm{~Hz})$. Table 1 summarizes data split and characteristics.

Table 1. Datasets

\begin{tabular}{|c|c|c|c|}
\hline & Training & Testing & Trajectory characteristics \\
\hline \multirow{2}{*}{$\begin{array}{c}\text { Biopsym } \\
\text { trajectory }\end{array}$} & $\begin{array}{c}5 \text { trajectories } \mathrm{x} 67 \text { patients }= \\
\begin{array}{c}300 \text { samples for training } \\
13000 \text { samples for validation }\end{array}\end{array}$ & $\begin{array}{c}2 \text { trajectories } \mathrm{x} 3 \text { patients } \\
=680 \text { samples }\end{array}$ & $\begin{array}{c}\text { Where y is the rotation around the } \\
\text { probe axis; }(\mathrm{x}, \mathrm{y}) \text { is the US probe } \\
\text { plane and } \mathrm{z} \text { the out-of-plane axis }\end{array}$ \\
\hline
\end{tabular}

\subsection{Evaluation}

Evaluating model predictions and their precision require to quantitatively compare predictions to labels (ground truth rotation parameters of $T_{t}$ ). To accurately represent the registration quality, it is particularly useful to measure its impact on the organ of interest in which targets are located. Target Registration Error (TRE) is the most frequently used metrics in image registration, because of its simplicity, fast computation, and ability to compare to most of the other studies in literature. This error is computed as defined in Equation (1). It is usually based on point pairs, most often fiducial landmarks manually defined by experts to establish the correspondence between two images. In the presented study, as a prostate surface mesh is associated to each $3 \mathrm{D}$ volume of the database, we used prostate mesh points (far more numerous than internal fiducials) to compute a robust TRE. According to the data generation (see Part 3.1), the simulator's reoriented volumes and their meshes are saved during each slice generation. Being given prostate mesh points $\left(p_{i}^{2 D}\right)$ of such reoriented volumes, we compute the TRE between these resulting points after network's predicted registration $\hat{T}_{t}\left(p_{i}^{2 D}\right)$ and the corresponding initial mesh points of the reference volume $\left(p_{i}^{3 D}\right)$ ( see Figure 3 ).

$$
T R E(m m)=\sqrt{\frac{\sum_{i=1}^{n}\left\|p_{i}^{3 D}-\hat{T}_{t}\left(p_{i}^{2 D}\right)\right\|^{2}}{n}}
$$


Since the TRE is a root mean square error, it gives an estimate of the standard deviation of the normal distribution of biopsy targets. To respect the confidence interval of $5 \mathrm{~mm}$ radius (clinical requirements cf 1.1), the TRE must be smaller than half of this value.

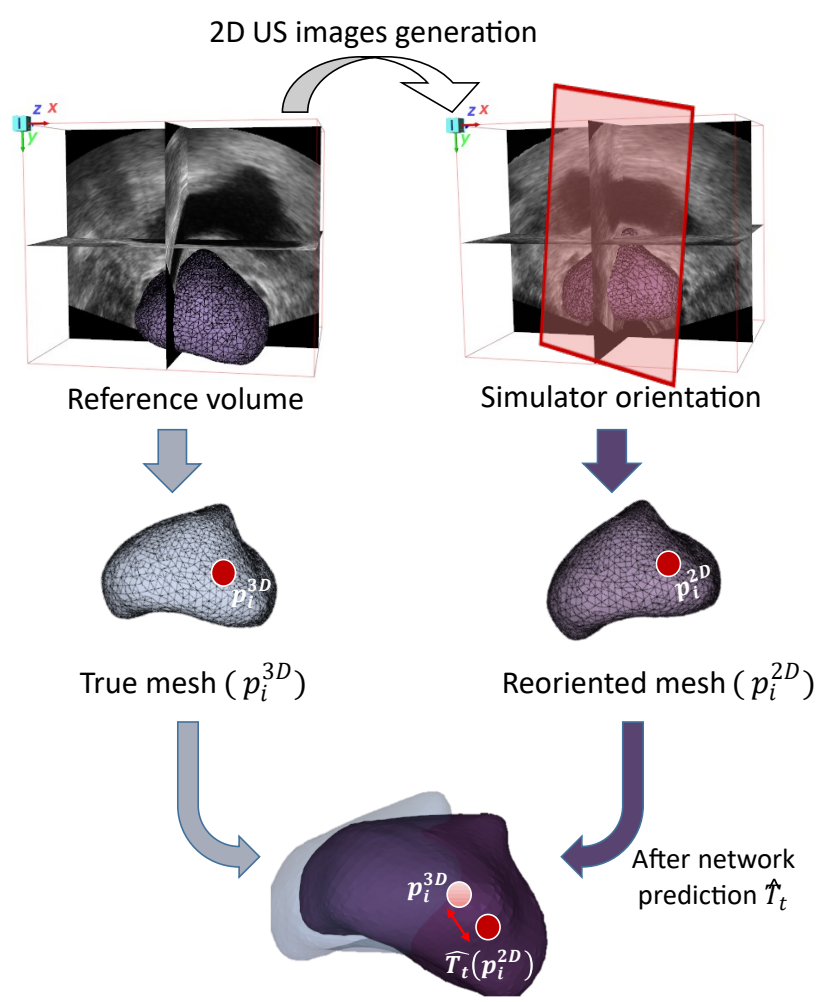

Figure 3. TRE-based evaluation using meshes: Reference mesh and reoriented mesh associated with the current US slice.

\subsection{Results and discussion}

Table 2 shows mesh-based TRE values before and after registration regarding the 4 scenarii in the presence of prior or not. The method with prior information outperforms the others, both in terms of accuracy and reliability. Scenario 3, which adds relative probe tracking information, achieves a mean TRE of $2.21 \mathrm{~mm} \pm 2.89$. These results meet clinical requirements (cf 1.1): with a response time of $28 \pm 4 \mathrm{~ms}$ (below $50 \mathrm{~ms}$ ) averaged on all test samples, and a TRE below $2.5 \mathrm{~mm}$. They are even more promising considering that the TRE value before any registration corresponds to large initial displacements $(\sim 30 \mathrm{~mm})$, for which a classical registration method would fail. Furthermore, testing data includes only unknown trajectories on unknown 3D US patient volumes, which also demonstrates efficient generalization capabilities of the network.

Figure 4-A illustrates the ground truth and predicted angles evolution over a trajectory, according to the 4 scenarii in the presence of prior, while Figure 4-B displays the associated TRE evolution over the same trajectory. It shows a good trajectory reconstruction without any drift Finally, our results highlight the usefulness of exploiting trajectory information especially through prior registration results and probe tracking parameters. In terms of computing time, the proposed method is able to perform $2 \mathrm{D} / 3 \mathrm{D}$ registrations in real-time and can be successfully used for continuous tracking during a biopsy exam. 
Table 2. Summary of the results obtained: Comparison with or without prior information, and comparison across the different scenarii. Scenario 1: pair of images only; scenario 2: pair of images + optical flow; scenario 3: pair of images + probe tracking information; scenario 4: pair of images with optical flow and probe tracking information

\begin{tabular}{|c|c|c|c|c|c|}
\hline$(\mathrm{mm})$ & TRE before & $\begin{array}{c}\text { TRE after } \\
\text { Scenario 1 }\end{array}$ & $\begin{array}{c}\text { TRE after } \\
\text { Scenario 2 }\end{array}$ & $\begin{array}{c}\text { TRE after } \\
\text { Scenario 3 }\end{array}$ & $\begin{array}{c}\text { TRE after } \\
\text { Scenario 4 }\end{array}$ \\
\hline Without $T_{\text {prior }}$ & $30.41( \pm 19.05)$ & $8.36( \pm 4.67)$ & $10.88( \pm 6.54)$ & $9.02( \pm 5.50)$ & $10.37( \pm 5.62)$ \\
\hline With $T_{\text {prior }}$ & idem & $4.36( \pm 5.16)$ & $4.03( \pm 5.4)$ & $2.21( \pm 2.89)$ & $2.60( \pm 3.22)$ \\
\hline
\end{tabular}
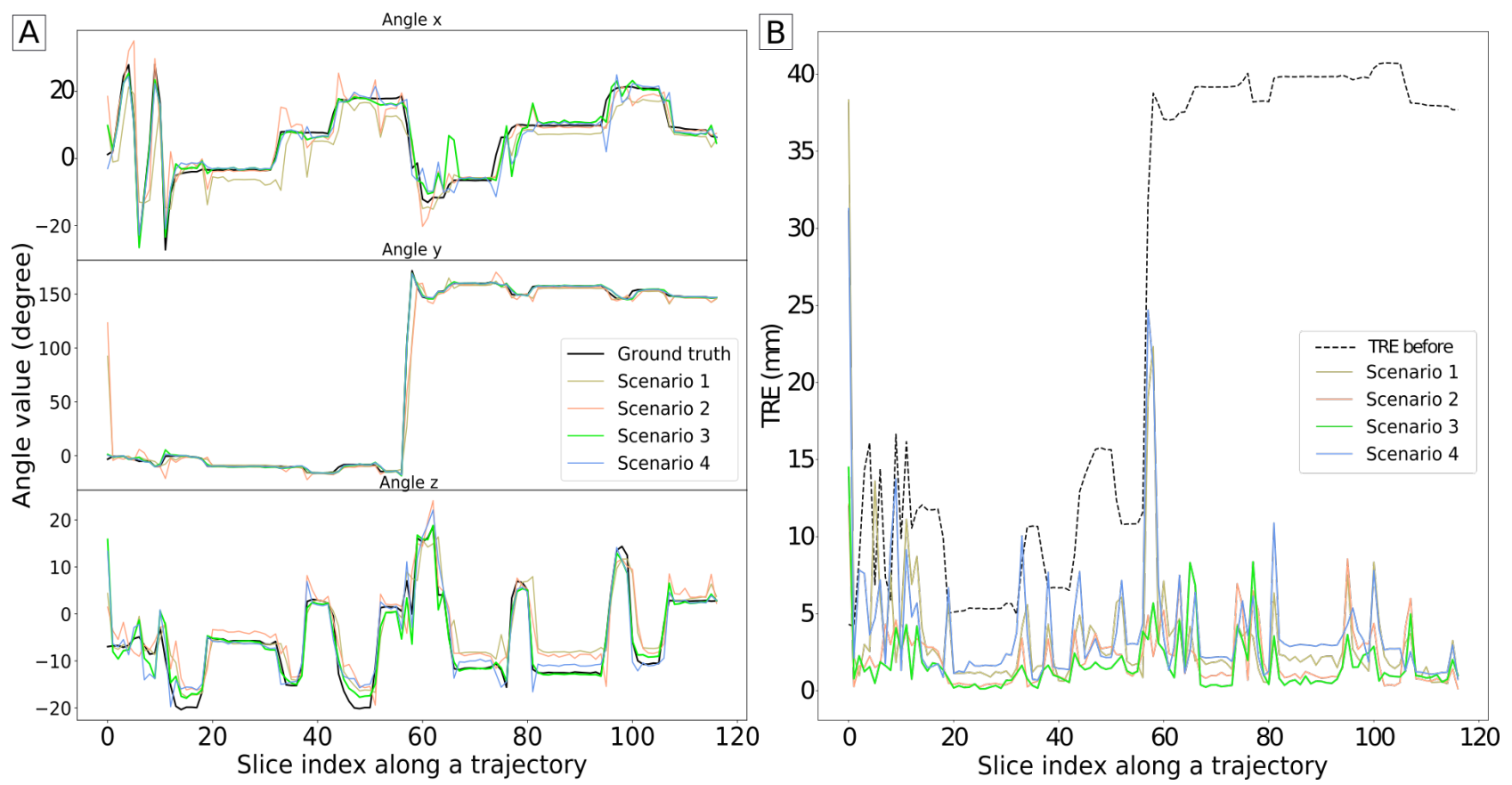

Figure 4. Temporal analysis over a complete trajectory, with time step between successive slices of $150 \mathrm{~ms}$. A) Angle evolution: Ground truth (black curve) and predicted angles evolution w.r.t the 4 scenarios in the presence of prior. B) TRE evolution

\section{CONCLUSION}

We developed a CNN to estimate the rotation parameters of a 2D/3D US registration transform in real-time. By adding trajectory information based on previous registration and probe tracking, we observe significant improvement of the registration quality. The network demonstrates efficient generalization capabilities on new patients and new trajectories. These results are comparable to related studies and achieve clinical requirements in terms of accuracy and computation time. They are even more promising considering that (i) input images are not preprocessed (only cropped) compared to other studies, (ii) US images present lower image quality than MRI images more often treated in the literature, (iii) complex and large displacements are tested as they are simulated in a realistic way from real biopsy procedure.

However, we constructed the database in an artificial and simplified way because of some simulator limitations, which did not take into account the complexity of real cases. For now, motions do not include translation and 2D images do not include possible deformation of the organ. Moreover, 2D images do not present realistic US noise patterns as they are all extracted from the same reference volume. Further improvements will include the generation of new databases from data collected during real clinical biopsy procedure in response to these issues.

Moreover, this method is limited to static analysis, with only one previous step consideration. Since the navigation assistance is a global temporal process and we have just demonstrated the benefit of prior trajectory consideration, the next step will be to investigate the potential benefit of recurrent network to retain the global 
navigation trajectory. With the hypothesis of stereotyped trajectories between each biopsy procedure, such dynamical analysis could be even more promising.

\section{ACKNOWLEDGMENTS}

Sincere thanks to clinicians from the Urology department of the Grenoble University Hospital, for their collaboration in data acquisition. This work was partly supported by the French Agence Nationale de la Recherche, "Investissement d'Avenir" program (grants MIAI@Grenoble Alpes under reference ANR-19-P3IA-0003 and CAMI Labex under reference ANR-11-LABX-0004)

\section{REFERENCES}

[1] Gillies, D. J., Gardi, L., De Silva, T., Zhao, S.-r., and Fenster, A., "Real-time registration of 3D to 2D ultrasound images for image-guided prostate biopsy," 44(9), 4708-4723.

[2] Karnik, V. V., Fenster, A., Bax, J., Cool, D. W., Gardi, L., Gyacskov, I., Romagnoli, C., and Ward, A. D., "Assessment of image registration accuracy in three-dimensional transrectal ultrasound guided prostate biopsy: Image registration accuracy in 3D TRUS-guided prostate biopsy," 37(2), 802-813.

[3] Cornud, F., Brolis, L., Delongchamps, N. B., Portalez, D., Malavaud, B., Renard-Penna, R., and Mozer, P., "TRUS-MRI image registration: A paradigm shift in the diagnosis of significant prostate cancer," 38(6), 1447-1463.

[4] Brown, A. M., Elbuluk, O., Mertan, F., Sankineni, S., Margolis, D. J., Wood, B. J., Pinto, P. A., Choyke, P. L., and Turkbey, B., "Recent advances in image-guided targeted prostate biopsy," 40(6), 1788-1799.

[5] Xu, S., Kruecker, J., Turkbey, B., Glossop, N., Singh, A. K., Choyke, P., Pinto, P., and Wood, B. J., "Real-time MRI-TRUS fusion for guidance of targeted prostate biopsies," 13(5), 255-264.

[6] Zeng, Q., Fu, Y., Tian, Z., Lei, Y., Zhang, Y., Wang, T., Mao, H., Liu, T., Curran, W. J., Jani, A. B., Patel, P., and Yang, X., "Label-driven magnetic resonance imaging (MRI)-transrectal ultrasound (TRUS) registration using weakly supervised learning for MRI-guided prostate radiotherapy," 65(13), 135002.

[7] Zeng, Q., Fu, Y., Jeong, J. J., Lei, Y., Wang, T., Mao, H., Jani, A. B., Patel, P., Curran, W. J., Liu, T., and Yang, X., "Weakly non-rigid MR-TRUS prostate registration using fully convolutional and recurrent neural networks," in [Medical Imaging 2020: Image Processing], Landman, B. A. and Išgum, I., eds., 105, SPIE.

[8] Zhu, N., Najafi, M., Han, B., Hancock, S., and Hristov, D., "Feasibility of Image Registration for UltrasoundGuided Prostate Radiotherapy Based on Similarity Measurement by a Convolutional Neural Network," 18, 153303381882196.

[9] Hu, Y., Modat, M., Gibson, E., Ghavami, N., Bonmati, E., Moore, C. M., Emberton, M., Noble, J. A., Barratt, D. C., and Vercauteren, T., "LABEL-DRIVEN WEAKLY-SUPERVISED LEARNING FOR MULTIMODAL DEFORMABLE IMAGE REGISTRATION," 5.

[10] Guo, H., Kruger, M., Xu, S., Wood, B. J., and Yan, P., "Deep adaptive registration of multi-modal prostate images," 101769.

[11] Baumann, M., Mozer, P., Daanen, V., and Troccaz, J., "Prostate biopsy tracking with deformation estimation," 16(3), 562-576.

[12] Selmi, S.-Y., Promayon, E., and Troccaz, J., "Hybrid 2D-3D ultrasound registration for navigated prostate biopsy," 13(7), 987-995.

[13] Tessier, D., Gillies, D. J., Gardi, L., Mercado, A., and Fenster, A., "Ring navigation: An ultrasoundguided technique using real-time motion compensation for prostate biopsies," in [Medical Imaging 2018: Image-Guided Procedures, Robotic Interventions, and Modeling], Webster, R. J. and Fei, B., eds., 52, SPIE.

[14] De Silva, T., Fenster, A., Cool, D. W., Gardi, L., Romagnoli, C., Samarabandu, J., and Ward, A. D., "2D-3D rigid registration to compensate for prostate motion during 3D TRUS-guided biopsy: 2D-3D rigid registration to compensate for prostate motion," $\mathbf{4 0}(2), 022904$.

[15] Beitone, C., Fiard, G., and Troccaz, J., "Towards real-time free-hand biopsy navigation," mp.14582.

[16] Hou, B., Alansary, A., McDonagh, S., Davidson, A., Rutherford, M., Hajnal, J. V., Rueckert, D., Glocker, B., and Kainz, B., "Predicting Slice-to-Volume Transformation in Presence of Arbitrary Subject Motion." 
[17] Yu, T.-F., Liu, P., Peng, Y.-L., Liu, J.-Y., Yin, H., and Liu, D. C., "Slice Localization for Three-Dimensional Breast Ultrasound Volume Using Deep Learning,"

[18] Mohseni Salehi, S. S., Khan, S., Erdogmus, D., and Gholipour, A., "Real-Time Deep Pose Estimation With Geodesic Loss for Image-to-Template Rigid Registration," 38(2), 470-481.

[19] Almogadwy, B., McLeod, K., and Burger, A., "A Deep Learning Approach for Slice to Volume Biomedical Image Integration," in [Proceedings of the 2019 11th International Conference on Bioinformatics and Biomedical Technology], 62-68, ACM.

[20] Li, Y., Khanal, B., Hou, B., Alansary, A., Cerrolaza, J. J., Sinclair, M., Matthew, J., Gupta, C., Knight, C., Kainz, B., and Rueckert, D., "Standard Plane Detection in 3D Fetal Ultrasound Using an Iterative Transformation Network," in [Medical Image Computing and Computer Assisted Intervention - MICCAI 2018], Frangi, A. F., Schnabel, J. A., Davatzikos, C., Alberola-López, C., and Fichtinger, G., eds., Lecture Notes in Computer Science 11070, 392-400, Springer International Publishing.

[21] Chen, Z., Xu, Z., Yi, W., Yang, X., Hou, W., Ding, M., and Granichin, O., "Real-time and multimodal brain slice-to-volume registration using CNN," 133, 86-96.

[22] Singh, A., Salehi, S. S. M., and Gholipour, A., "Deep Predictive Motion Tracking in Magnetic Resonance Imaging: Application to Fetal Imaging," 1-1. Comment: The article has been published in IEEE TMI: 14 pages, 11 figures, 2 tables and 1 supplementary https://github.com/bchimagine/DeepPredictiveMotionTracking.

[23] Miao, S., Wang, Z. J., and Liao, R., "A CNN Regression Approach for Real-Time 2D/3D Registration," 35(5), 1352-1363.

[24] Miao, S., Wang, Z. J., Zheng, Y., and Liao, R., "Real-time 2D/3D registration via CNN regression," in [2016 IEEE 13th International Symposium on Biomedical Imaging (ISBI)], 1430-1434, IEEE.

[25] Bhardwaj, A., Park, J.-S., Mukhopadhyay, S., Sharda, S., Son, Y., Ajani, B. N., and Kudavelly, S. R., "Rigid and deformable corrections in real-time using deep learning for prostate fusion biopsy," in [Medical Imaging 2020: Image-Guided Procedures, Robotic Interventions, and Modeling], Fei, B. and Linte, C. A., eds., 61, SPIE.

[26] Wei, W., Rak, M., Alpers, J., and Hansen, C., "TOWARDS FUlLY AUTOMATIC 2D US TO 3D CT/MR REGISTRATION: A NOVEL SEGMENTATION-BASED STRATEGY," 6.

[27] Hu, Y., Modat, M., Gibson, E., Li, W., Ghavami, N., Bonmati, E., Wang, G., Bandula, S., Moore, C. M., Emberton, M., Ourselin, S., Noble, J. A., Barratt, D. C., and Vercauteren, T., "Weakly-supervised convolutional neural networks for multimodal image registration," 49, 1-13.

[28] Prevost, R., Salehi, M., Jagoda, S., Kumar, N., Sprung, J., Ladikos, A., Bauer, R., Zettinig, O., and Wein, W., "3D freehand ultrasound without external tracking using deep learning," 48, 187-202.

[29] Farnebäck, G., "Two-Frame Motion Estimation Based on Polynomial Expansion," in [Image Analysis], Bigun, J. and Gustavsson, T., eds., 2749, 363-370, Springer Berlin Heidelberg.

[30] Kok, M., Hol, J. D., and Schön, T. B., "Using Inertial Sensors for Position and Orientation Estimation," 11(1-2), 1-153. Comment: 90 pages, 38 figures.

[31] Selmi, S.-Y., Fiard, G., Promayon, E., Vadcard, L., and Troccaz, J., "A virtual reality simulator combining a learning environment and clinical case database for image-guided prostate biopsy," in [26th IEEE International Symposium on Computer-Based Medical Systems, CBMS'2013], 179-184, IEEE.

[32] Fiard, G., Selmi, S.-Y., Promayon, E., Descotes, J.-L., and Troccaz, J., "Simulation-based training for prostate biopsies: Towards the validation of the Biopsym simulator," 1-7. 